\title{
O transtorno factício das síndrome de Munchausen e síndrome de Munchausen por Procuração: uma revisão narrativa de literatura
}

\author{
The factitious disorder of Munchausen Syndrome and Munchausen Syndrome by Proxy: a \\ narrative literature review
}
El trastorno facticio del síndrome de Munchausen y el síndrome de Munchausen por poder: una revisión de la literatura narrativa

Izabela Machado Gonçalves ${ }^{1 *}$, Tiago de Castro Pimentel ${ }^{2}$, Rafaela Salomão Moura ${ }^{1}$, Bianca da Rocha Siqueira ${ }^{1}$, Felipe de Castro Pimentel ${ }^{3}$, Victor Lucas Ferreira ${ }^{1}$, Sebastião Jorge da Cunha Gonçalves ${ }^{1}$.

\section{RESUMO}

Objetivo: Analisar a Síndrome de Munchaunsen (SM) e a Síndrome de Munchaunsen por Procuração (SMP). Revisão bibliográfica: A SM ocorre quando o individuo produz sinais e sintomas de uma doença ou lesão causando dano ao seu corpo, sem intenção de ganho secundário. A SMP é uma forma específica de abuso infantil que retrata crianças das quais os responsáveis fabulavam histórias de doenças sobre seu filho e alimentavam as histórias criadas com falsos sinais físicos, sendo aplicada a crianças que são impostas a simular as sintomatologias empregadas pelo responsável. Considerações finais: Pacientes com estas síndromes despendem recursos e tempo das equipes de saúde em decorrência de avaliações e procedimentos não necessários. Tais síndromes podem ser associadas com iatrogênese caso o distúrbio factício não seja percebido pelos profissionais de saúde. Dessa forma, é essencial o preparo da equipe de saúde para reconhecer, gerenciar e tratar essas síndromes fazendo a correta abordagem multiprofissional de modo a oferecer os devidos cuidados ao paciente.

Palavras-chave: Síndrome de Munchausen, Síndrome de Munchausen causada por terceiro, Violência.

\begin{abstract}
Objective: To analyze Munchaunsen Syndrome (SM) and Munchaunsen syndrome by Proxy (SMP). Bibliographic review: MS occurs when the individual produces signs and symptoms of a disease or injury causing damage to his body, with no intention of secondary gain. SMP is a specific form of child abuse that portrays children whose guardians made up stories of illnesses about their child and fed the stories created with false physical signs, being applied to children who are forced to simulate the symptoms employed by the guardian. Final considerations: Patients with these syndromes consume resources and time from the healthcare teams that assist them, due to unnecessary assessments and procedures that may be associated with iatrogenesis if the factitious disorder is not detected by healthcare professionals. Thus, it is essential to prepare the health team to recognize, manage and treat these syndromes, taking the correct multidisciplinary approach in order to provide care without judgment.
\end{abstract}

Keywords: Munchausen syndrome, Munchausen syndrome by proxy, Violence.

\footnotetext{
1 Universidade de Vassouras (UV), Vassouras - RJ.*E-mail: izabela-machado@hotmail.com

2 Universidade Iguaçu (UNIG CAMPUS IV), Itaperuna - RJ.

${ }^{3}$ Universidade Federal do Espírito Santo (UFES), Vitória - ES.
}

SUBMETIDO EM: 10/2021

ACEITO EM: 10/2021

PUBLICADO EM: 11/2021 


\section{RESUMEN}

Objetivo: Analizar el Síndrome de munchaunsen (SM) y el Síndrome de munchaunsen por proxy (SMP). Revisión bibliográfica: La EM ocurre cuando el individuo produce signos y síntomas de una enfermedad o lesión que causa daño a su cuerpo, sin intención de obtener beneficios secundarios. SMP es una forma específica de abuso infantil que retrata a niños cuyos tutores inventaron historias de enfermedades sobre su hijo y alimentaron las historias creadas con señales físicas falsas, siendo aplicada a niños que se ven obligados a simular los síntomas empleados por el tutor. Consideraciones finales: Los pacientes con estos síndromes consumen recursos y tiempo de los equipos sanitarios que les asisten, debido a valoraciones y procedimientos innecesarios que pueden estar asociados a la iatrogénesis si el trastorno facticio no es detectado por los profesionales sanitarios. Por ello, es fundamental preparar al equipo de salud para reconocer, gestionar y tratar estos síndromes, adoptando el correcto abordaje multidisciplinario para poder brindar una atención sin juzgar.

Palabras clave: Síndrome de Munchausen, Síndrome de Munchausen causado por tercero, Violencia.

\section{INTRODUÇÃO}

O conceitoSíndrome de Munchausen (SM) foi adotado por Asher em 1951a fim de caracterizar indivíduos que produzem de forma intencional sintomas físicos para receber assistência médica ou hospitalar carecendo de longas investigações e intervenções, sem ganhos secundários (LIMA KRF, et al., 2019; SIMÃO JSS, et al., 2020; SOUSA FILHO D, et al., 2017; PAVEZ MV, et al., 2016).

Em 1977, Meadow adotou o termo Síndrome de Munchausen por procuração (SMP) para descrever quando o portador do transtorno produz ou simula os sintomas em outra pessoa incapaz de se defender, como por exemplo, em uma criança, a fim de requerer atenção das outras pessoas. Em cerca de $90 \%$ dos casos da (SMP), o perpetrador é a mãe da criança (SIMÃO JSS, et al., 2020; SOUSA FILHO D, et al., 2017). O comportamento da mãe agressora simula sentimentos de preocupação e devoção pelo filho, o que dificulta o diagnóstico, havendo casos em que a criança participa simbioticamente dessa situação (GONCALVES TG, et al., 2014).

Os transtornos factícios são de difícil diagnóstico pela forma com que o portador no caso da SM ou do perpetrador no caso da SMP se comportam mediante a equipe de saúde, ou seja, a maneira com a qual transmitem a veracidade dos fatos. No caso da SMP, muitas vezes, existe a ajuda da criança para a confirmação da história. Estes transtornos são evitáveis por meio da busca da história pregressa, verificação dos sinais e sintomas e dos exames laboratoriais (LIMA KRF, et al., 2019).

Tanto a SMP quanto a SM são transtornos fictícios, que se caracterizam pela simulação de sintomas coerentes, cujo objetivo é assumir o papel de doente, por meio da manipulação de sinais e sintomas (SIMÃO JSS, et al., 2020). São síndromes de difícil diagnóstico que representam um grande desafio para os serviços e profissionais de saúde, seja pela iatrogenia que podem causar e pelos altos custos ao sistema de saúde (SOUSA FILHO D, et al., 2017).

O objetivo do estudo foi analisar as características da síndrome de munchaunsen e da sindrome de munchaunsen por procuração.

\section{REVISÃO BIBLIOGRÁFICA}

\section{Síndrome de Munchausen}

A SM ocorre quando o individuo elabora sinais e sintomas de uma doença ou lesão acarretando prejuízo ao seu corpo. Traduz-se em mentiras elaboradas, narrativas de vários problemas médicos comhistória inconsistente e comportamento alterado. Ademais, há a obrigação psicológica em adotar o papel de doente sem haver ganhos ou incentivos evidentes. Dessa maneira, procura atendimento médico recorrente, submetendo-se à internação e às vezes a intervenções médicas invasivas e até mesmo procedimentos cirúrgicos importantes. O transtorno está associado a sofrimento psicológico e prejuízo funcional, e a gravidade da doença varia de leve a grave (GARCIA NP, et al., 2019; BERUTTI LB, et al., 2020). 
O estímulo para este comportamento é incerto porque esses pacientes não buscam a aquisição de benefícios diretos. O comportamento é usado para procurar tratamento médico, atingindo as internações hospitalares e procedimentos médicos invasivos. O transtorno está associado a sofrimento psicológico e prejuízo funcional, e a gravidade da doença varia de leve a grave (GARCIA NP, et al., 2019).

A SM pode se manifestar de maneira isolada ou concomitante a outros distúrbios psiquiátricos tais quais esquizofrenia, depressão, transtornos de ansiedade e de personalidade Border-line. O diagnóstico diferencial inclui transtornos somatoformes, no qual existe uma doença se houver uma causa orgânica aparente e que não é produzida de forma consciente e nem sustentada por informaçãoes fictícias pelo paciente. A simulação acontece quando o paciente produz sinais e sintomas, reproduzindo uma doença, mas com o propósito de adquirir algum ganho pessoal (BERUTTI LB, et al., 2020).

A etiologia do transtorno factício imposto a si mesmo é desconhecida. Este está associado a fatores psicossociais, comprometimento neurocognitivo e anormalidades de neuroimagem que podem talvez contribuir para a patogênese. Existem algumas teorias quanto à patogênese da síndrome como a de que há uma disfunção no hemisfério direito, o que foi identificado por estudo com 5 pacientes nos quais a inteligência estava superficialmente intacta $e$ as habilidades verbais eram excelentes, mas havia desorganização conceitual e prejuízo do gerenciamento de informações complexas e do julgamento (GONÇALVES BM, et al., 2020; WITTKOWSKI H, et al., 2017).

Há, ainda, hipóteses de que existam anormalidades no sistema nervoso central (focos epileptogênicos, lesões cranianas de diversas etiologias, infecções prévias, disfunções no eletroencefalograma; além de alterações no sistema hipotalâmico-pituitário-adrenal (GONÇALVES BM, et al., 2020).

A primeira manifestação do transtorno factício imposto a si mesmo ocorre, geralmente, na terceira ou quarta década de vida, entretanto, crianças já foram identificadas com a síndrome. O início normalmente acontece após uma hospitalização por causa de uma condição médica geral ou transtorno mental, e a SM parece se desenvolver gradualmente (BASS C e LASER D, 2014; GONÇALVES BM, et al., 2020).

Em geral o portador da síndrome possui algum tipo de experiência ou contato com a àrea da saúde. Além disso, possui, também, traços de personalidade antissocial. O paciente passa por diversos hospitais, sendo, dessa forma, várias vezes investigado. Descreve-se também que essas múltiplas passagens por instituições podem ser mais um elemento fantasioso da doença. Muitas vezes, encontra-se evidência física da autoindução dos sinais (GONÇALVES BM, et al., 2020).

O curso normal da doença parece ser um de episódios intermitentes, embora episódios únicos, bem como episódios crônicos e ininterruptos também sejam relatados. A recuperação a longo prazo do transtorno factício pode ocorrer, mas parece ser rara. O transtorno factício ocorre mais frequentemente em mulheres, indivíduos solteiros, profissionais de saúde (antigos ou presentes), história psiquiátrica passada, conflito familiar (por exemplo, abuso ou divórcio dos pais). Outros fatores de risco para transtorno factício incluem idade $\leq 40$ anos, história de trauma e história psiquiátrica familiar (BASS C e GLASER D, 2014; KOZLOWSKA K, 2014).

A SM está frequentemente relacionada a fatores psicossociais que incluem perdas prematuras por morte, doença ou abandono; vínculos interrompidos com outras pessoas devido negligência, abuso, institucionalização ou outros traumas; experiências reforçadoras gratificantes relacionadas ao papel de doente; e um desejo de atenção. A perspectiva do desenvolvimento postula que os comportamentos anormais da doença começam na infância como um mecanismo para lidar com o estresse (BASS C e GLASER D, 2014; GARCIA NP, et al., 2019).

O comportamento de papel de doente associado ao transtorno factício é uma maneira de estabelecer ou estabilizar a identidade de alguém, manter relacionamentos com outras pessoas e lidar com problemas emocionais e necessidades não supridas. Doenças crônicas e/ ou hospitalização podem oferecer aos pacientes benefícios que de outra forma não estariam disponíveis em ambientes sociais mais convencionais como um papel claro no ambiente em que eles estão inseridos além de sensação de importância, pertencimento, preocupação e aceitação por outros (BASS C e GLASER D, 2014; WITTKOWSKI H, et al., 2017). 
Quanto às motivações que fazem o paciente buscar atenção médica, a maioria deles possui um comportamento alterado, sentindo uma obrigação psicológica em se posicionar como doente sem, apesar disso, ter ganhos ou incentivos aparentes. Ademais, há uma necessidade de atenção que o coloca na posição de paciente para ser admitido em hospitais e uma história pregressa de abuso infantil, traumas, privação afetiva e experiências de perda durante a infância. $O$ hospital se torna o local onde o paciente encontra o acolhimento e atenção desejados (GARCIA NP, et al., 2019).

Em geral, o paciente já passou por diversos hospitais e se "dá alta" apesar das orientações médicas, após conflitos e discussões com médicos e enfermeiros, e em seguida busca atendimento em outros locais. Muitos pacientes utilizam histórias falsas além da doença e dos sintomas fictícios, inventando novos nomes, endereços e profissões, impossibilitando o acesso aos registros médicos e dificultando o diagnóstico (GARCIA NP, et al., 2019). Poucos pacientes recebem o devido atendimento psiquiátrico. A maioria deles é visto nos serviços de emergência, sendo o diagnóstico complicado pelo desconhecimento dos profissionais de saúde sobre a SM(WITTKOWSKI H, et al., 2017).

A avaliação de pacientes cuja suspeita diagnóstica é de SM consiste na avaliação do histórico médico e psiquiátrico geral, exame do estado físico e mental além de uma análise detalhada da cronologia do registro médico. Ademais, informações colaterais de familiares ou amigos e de outros registros podem ser úteis. Apesar dos testes laboratoriais poderem demonstrar evidências de comportamento factício, não existe um teste diagnóstico específico para o transtorno factício, sendo essencial considerar a confiabilidade do parecer médico no diagnóstico (VECINA MC e JIMÉNES-PERIANES A, 2020).

O diagnóstico pode ser feito através dos critérios do Manual Diagnóstico e Estatístico de Transtornos Mentais (DSM-5), que requerem cada uma das seguintes características: adulteração de sinais ou sintomas físicos ou mentais, ou indução de ferimento ou doença, associada ao engano identificado; o indivíduo se mostra aos outros como enfermo, debilitado ou ferido; a conduta enganosa é evidente mesmo na inexistência de recompensas externas óbvias; o comportamento não é melhor esclarecido por outro distúrbio mental, como transtorno delirante ou outro transtorno psicótico. O diagnóstico de transtorno factício requer a identificação objetiva de comportamentos de falsificação da doença e evidências de engano, ao invés de inferência sobre a intenção ou possível motivação subjacente (AMERICAN PSYCHIATRIC ASSOCIATION (APA), 2014).

O tratamento é baseado primordialmente em psicoterapa individual ou de grupo de apoio ou terapia cognitivo-comportamental é frequentemente sugerida. Além disso, são essenciais medidas de suporte físico, psicológico e social. Deve-se buscar a motivação para o transtorno e de forma a consciencializar o paciente de que aquilo pode ser prejudicial a ele além de ofertar conforto e segurança para tentar chegar à resolução dos problemas psicológicos e evitar recitavas (GONÇALVES BM, et al., 2020; VECINA MC e IMÉNESPERIANES A, 2020).

Para o tratamento pode ser necessária a utilização de antidepressivos e antipsicóticos. Não é em todos os casos que o confronto com o paciente em relação a suas ações e doença deve ser realizado. Quando isso acontecer, deve ser feito por profissional treinado para esse tipo de abordagem, pois pode gerarnegação no paciente com consequente evasão do hospital ou tratamento (GONÇALVES BM, et al., 2020).

O prognóstico para transtorno factício imposto a si mesmo é ruim e a recuperação parece ser infrequente, especialmente entre os pacientes que são identificados posteriormente em seu curso (GONÇALVES BM, et al., 2020; SCHRADER H, et al., 2017). O transtorno factício pode levar a procedimentos diagnósticos e terapêuticos que resultam em morbidade irreversível (SCHRADER $\mathrm{H}$, et al., 2017).

As mortes também podem ocorrer porque os pacientes subestimam a letalidade de seu comportamento; uma série de casos de 20 pacientes descobriu que o comportamento artificial levou diretamente à morte de 20\% (SCHRADER H, et al., 2017). O suicídio também pode ocorrer, mas não está claro com qual frequência ocorre (BASS C e GLASER D, 2014). 


\section{Síndrome de Munchausen por procuração}

Conforme a sociedade foi se tornando mais sensível às consequências dos maus tratos de crianças e adolescentes, a violência intrafamiliar, que até então era legitimada por um imaginário social da violência como estratégia disciplinadora e como forma de se resolver conflitos, passou a ser alvo de atenção de profissionais da saúde e educação (TACHIBANA M e FERREIRA GD, 2020). Violência contra a criança consiste em violação dos direitos humanos e representa grave problema de saúde pública. A Organização Mundial da Saúde (OMS) estima que um bilhão de crianças menores de 17 anos de idade já foi vítima de algum tipo de abuso (MIZIARA CSMG, et al., 2020).

A SMP é uma forma grave e específica de abuso infantil, descrita por Meadow em 1977, a fim de caracterizar crianças cujos responsáveis inventavam e alimentavam histórias de doenças criadas com falsos sinais físicos. O termo "por procuração" retrata o responsável que fornece informação falsa e simula ou estimula sintomas na criança (LIMA KRF, et al., 2019; RODRIGUEZ LB, et al., 2018).

É comum que esforços excessivos e malsucedidos sejam feitos para individualizar um diagnóstico que explique os sintomas que o paciente apresenta. A busca sem respostas que expliquem a origem dos sintomas induz um zelo clínico que obscurece o bom julgamento médico. Além disso, a condição normalmente parece resistente a qualquer tipo de tratamento (SCHRADER $\mathrm{H}$, et al., 2017).

A síndrome de Munchausen causada por terceiro ou transtorno factício imposto a outro é classificada na categoria T74.8 da Classificação Internacional de Doenças (CID 10), outras síndromes especificadas de maus-tratos. O termo também é usado para caracterizar idosos ou indivíduos com deficiência e/ou adultos dependentes, nos quais os sintomas e sinais são criados e possuem exames alterados por seus cuidadores (SOUSA FILHO D, et al., 2017).

É uma doença de difícil controle que quando não diagnosticada e tratada a tempo pode levar ao óbito (FERRÃO ACF, NEVES MGC, 2013). Sua incidência é de aproximadamente 0,5 a 2,0 por 100.000 crianças menores de 16 anos, entretanto existe subnotificação pela identificação não ser realizada de forma correta. Pode ocorrer em ambos os sexos com maior incidência em crianças menores (LIMA KRF, et al,, 2019).

A morbimortalidade da SMP decorre da ação direta do perpetrador e das intervenções médicas invasivas movidas pelas descrições do cuidador. A mortalidade geral é de $6 \%$ a $9 \%$, podendo chegar a $12 \%$ em crianças menores de 16 anos. Em adultos a taxa de mortalidade é desconhecida, porém existe um baixo índice de suspeita de SMP em idosos (LIMA KRF, et al., 2019).

Quanto a sua apresentação clínica, deve-se suspeitar de SMP particularmente quando os sinais e sintomas apresentados aparecem apenas na presença do cuidador ou são apenas presenciados por ele. A resposta ao tratamento instituído é anormalmente ruim e os sintomas mudam de padrão com frequência (NASCIMENTO RC, et al., 2017; MARANA AP, et al., 2018).

O cuidador exerce pressão para investigações adicionais a fim de chegar a um diagnóstico conclusivo, podendo levar, inclusive, o paciente a vários médicos. Há uma limitação do paciente desproporcional à doença diagnosticada e o quadro clínico e a evolução não são bem explicados por um diagnóstico, ainda que raro (NASCIMENTO RC, et al., 2017; MARANA AP, et al., 2018). Ao avaliar mais apuradamente o comportamento da mãe, a equipe médica percebe que ela está mais interessada em obter a atenção do médico do que nos próprios sinais e sinais da paciente (GONÇALVES BM, et al., 2020).

Em geral, os perpetradores conhecem variadas informações médicas e possuem bom relacionamento com a equipe assistencial. A presença de uma mãe perpetradora superprotetora e ausência da figura paterna são típicas da doença. O perfil psicológico dos cuidadores do paciente e a história social também são úteis na formulação da suspeita da síndrome (NASCIMENTO RC, et al., 2017; MARANA AP, et al., 2018).

Na maior parte dos casos, as mães dos agressores são mulheres com idades entre 20 e 30 anos que tendem a ser mães calmas e que demonstram confiança nos médicos a fim de obter sua atenção. Ademais, observa-se que a relação estabelecida pela mãe com o filho tem uma característica excessiva (WITTKOWSKI H, et al., 2017) 
Os principais sintomas que o perpetrador produrz na criança são: hemorragia induzida, convulsões, apneia, diarreia, vômitos e febre além de hipoglicemia, dor abdominal e infeção (MANTAN M et al, 2015; SILVA HM, PRISZKULNIK L, 2013). Entretanto, não é em todos os casos que o perpetrador manipula o corpo da criança induzindo verdadeiramente os sintomas (BROWN AN et al., 2014). Alguns perpetradores colocam de forma falsa a criança na condição de doente a ser diagnosticada e investigada baseada em relatos fantasiosos até aqueles que adulteram os dados de exames clínicos da criança ou, nos casos mais graves, os que induzem a doença de fato(MANTAN M, et al., 2015; SILVA HM e PRISZKULNIK L, 2013; BROWN NA, et al., 2014).

Essas ações servem de critério para a identificação da gravidade do caso, uma vez que relatos falsos possuem menor gravidade que a modificação de exames laboratoriais, que, por sua vez, são menos comprometedores que a efetiva perpetração de sintomas corporais (SCHRADER $\mathrm{H}$, et al., 2017)

As mães perpetradoras são agradáveis, cooperativas com os procedimentos e apreciam o trabalho dos médicos. Estão aparentemente preocupadas e são afetuosas com os filhos doentes, porém não demonstram tanta preocupação quanto à possível causa da doença. Destaca-se a tendência do perpetrador de se sentir especial por possuir um "caráter heróico" no que diz respeito ao cuidado com as crianças doentes, chamando, dessa maneira, atenção da família, amigos e comunidade (VECINA MC e JIMÉNESPERIANES A, 2020)

Os perpetradores possuem motivações variadas que podem incluir desde uma extrema ansiedade que a faz exagerar os sinais e sintomas dos filhos como forma de encorajar o médico a excluir qualquer doença tratável até eximir-se da culpa pelas dificuldades comportamentais da criança ou manter relação de proximidade com o filho. Pode haver, também, uma tentativa de fuga de um casamento infeliz ou a aproximação com um marido ausente (BASS C e GLASER D, 2014).

A motivação principal na SMP é chamar a atenção de prestadores de serviços médicos na busca por assumir o papel de doente através da criança, a qual permite que o perpetrador fique no hospital junto de seu filho (BASS C e GLASER D, 2014). A necessidade de causar a doença no filho, geralmente, ocorre devido a uma perda precoce na vida da perpetradora a qual pode estar relacionada a uma rejeição materna e pelo sentimento de falta de amor e atenção (VECINA MC e JIMÉNES-PERIANES A, 2020)

Muitas mães que sofrem de síndrome de Munchausen por procuração foram vítimas de abuso na infância o qual envolveu privação, negligência e traumas. A indução da doença nos filhos pode funcionar como um meio para que a mãe trabalhe sua própria dinâmica de abuso precoce. A raiva reprimida por ter sido vítima na infância é encenada na criança, permitindo que ela busque ajuda sem ser necessário confrontar diretamente seu passado abusivo (SCHRADER H, et al., 2017)

O diagnóstico de SMP exige que o médico reconheça que o cuidador está exagerando, inventando ou induzindo a doença em uma criança. Identificar que a relação médico paciente normal se tornou patológica é fundamental para fazer o diagnóstico; o médico agora está prejudicando o paciente em vez de ajudá-lo por causa do engano do cuidador (FLAHERTY EG, et al., 2013).

A SMP pode ser classificada como leve, moderada ou grave e a identificação precoce de informações históricas distorcidas, com sorte antes que uma criança seja prejudicada, é a melhor maneira de evitar que a situação evolua para aumento da gravidade (APA, 2014).

O tratamento consiste em identificar o mais precocemente possível a SMP. A suspeita ou confirmação de MCA moderado ou grave justifica notificação dos serviços de proteção à criança e, sempre que possível, consulta com uma equipe multidisciplinar de abuso infantil liderada por um especialista em abuso infantil. A transferência para um centro de cuidados terciários pediátricos pode ser necessária para obter os recursos adequados para a criança. Assim que a SMP for identificada, os cuidados médicos desnecessários devem ser interrompidos (APA, 2014; VECINA MC e JIMÉNES-PERIANES A, 2020).

A maioria dos esforços de proteção à criança trabalha para manter as crianças com suas famílias, se isso puder ser feito com segurança. Para crianças vítimas de SMP, isso significa que elas não podem continuar a ser exposta a cuidados médicos desnecessários e aos danos iatrogênicos associados a eles; 0 perpetrador deve mudar suas crenças e comportamentos em relação à questões médicas (APA, 2014). 


\section{CONSIDERAÇÕES FINAIS}

A SM e a SMP são desafios para a medicina por serem transtornos factícios ligados à simulação de sinais e sintomas de uma doença em si ou a outros e estão associadas com alta morbimortallidade. Pacientes com estas síndromes despendem recursos e tempo das equipes de saúde em decorrência de avaliações, exames complementares e procedimentos não necessários. Nesse contexto, tais síndromes podem ser associadas com iatrogênesecaso o distúrbio não seja percebido pelos profissionais de saúde. Dessa forma, é essencial o preparo da equipe de saúde para reconhecer, gerenciar e tratar essas síndromes fazendo a correta abordagem multiprofissional de modo a oferecer os devidos cuidados ao paciente.

\section{REFERÊNCIAS}

1. AMERICAN PSYCHIATRIC ASSOCIATION (APA). Manual diagnóstico e estatístico de transtornos mentais: DSM-5. Porto Alegre: Artmed, 2014; 992p.

2. BASS C, GLASER D. Early recognition and management of fabricated or induced illness in children. The Lancet, 2014; 383: 1412-1421.

3. BERUTTI LB, et al. Manifestações orofaciais na síndrome de Munchausen - Revisão de literatura. Arq Med HospFacCienc Med Santa Casa São Paulo, 2020; 65: e40.

4. BROWN AN, et al. Caretaker blogs in caregiver fabricated illness in a child: a window on the caretaker's thinking? Child Abuse \& Neglect, Elmsford, 2014; 38: 488-497.

5. FERRÃO ACF, NEVES MGC. Síndrome de Munchausen por Procuração: quando a mãe adoece o filho. Com. Ciências Saúde. 2013; 24(2): 179-186.

6. FLAHERTY EG, et al. Caregiver-fabricated illness in a child: a manifestation of child maltreatment. Pediatrics. 2013;132(3):590.

7. GARCIA NP, et al. Síndrome de Munchausen: Um caso de confissão espontânea da simulação de sinais e sintomas. Colloq Vitae, 2019; 11(2): 52-58.

8. GONÇALVES BM, et al. Síndrome de Munchausen relacionada à calculose urinária não metabólica: um relato de caso. Revista de Patologia do Tocantins. 2020; 7(2): $41-44$.

9. GONCALVES TG, et al. Síndrome de Munchausenby proxy: definición, contextualización y factores psíquicos involucrados. Revista de Psicología, 2014, 32(1): 139-156.

10. KOZLOWSKA K. Abnormal illness behaviours: a developmental perspective. The Lancet 2014; 383:1368.

11. LIMA KRF, et al. Síndrome de Münchausen por Procuração: Revisão Integrativa. RevEnf Atual In Derme, 2019; 88(26): 1-8.

12. MANTAN M, et al. Acute kidney injury in a child: a case of Munchausen syndrome by Proxy. Saudi Journal of Kidney Diseases and Transplantation, Riyadh, 2015; 26(6): 1279-1281.

13. MARANA AP, et al. Munchausen por poderes: dificultades diagnósticas y terapéuticas. RevPediatr Aten Primaria, 2018, 20(80): 105-108.

14. MIZIARA CSMG, et al. Doença fabricada ou induzida pelos cuidadores: maus-tratos graves e silencioso. Saúde, Ética \& Justiça, 2020; 25(1): 28-31.

15. NASCIMENTO RC, et al. Síndrome de Munchausen e Transtorno Factício por Procuração: um Desafio para o Pediatra. RevCientHosp Santa Izabel. 2017; (1): 20-23.

16. PAVEZ MV et al. Síndrome de Münchausen por poder en otorrinolaringología. Rev. Otorrinolaringol. Cir. Cabeza Cuello, 2016; 76(2): 231-235.

17. RODRIGUEZ LB, et al. Lesiones orales en víctimas del síndrome de munchausen por poder. Med. leg. Costa Ric, 2018; 35(2) :37-44.

18. SCHRADER $\mathrm{H}$, et al. Challenges presented by Munchausen syndrome. Tidsskrift for den Norske Lægeforening. 2017; 137(10): 696- 697.

19. SIMÃO JSS, et al. Educação em saúde para agentes comunitários de saúde sobre a síndrome de Munchausen, um relato de experiência extensionista. Rev. Ciênc. Ext, 2020; 16: 243-250.

20. SILVA HM, PRISZKULNIK L. Síndrome de Munchausen por procuração, a Psicologia e a Psicanálise: conhecer para suspeitar. Psicología, Conocimiento y Sociedad, Montevideo, 2013, 3(2): 155-170.

21. SILVA NMR, et al. Dermatite factícia desencadeada pela síndrome de Münchausen. Anais Brasileiros de Dermatologia, 2010, 85:(1): 77-79.

22. SOUSA FILHO D, et al. Síndrome de Munchausen e síndrome de Munchausen por procuração: uma revisão narrativa. Einstein. 2017; 15(4): 516-21.

23. SUTHALL D, et al. Gravações de vídeo secretas de abuso infantil com risco de vida: Lições para a proteção da criança. Pediatrics 1997; 100: 735-60.

24. TACHIBANA M, FERREIRA GD. O cuidado materno violento: reflexões psicanalíticas sobre a Síndrome de Munchausen por Procuração. Semina: Ciências Sociais e Humanas, 2020, 41(2): 229-248.

25. VECINA MC, JIMÉNES-PERIANES A. Aproximación teórica al Síndrome de Munchausen por Poderes. Behavior\& Law Journal, 2020; 6(1): 1-11

26. WITTKOWSKI H, et al. Munchausen by proxy syndrome mimicking systemic autoinflammatory disease: case report and review of the literature. PediatrRheumatol Online J. 2017; 15(19): 1-5. 